\title{
Polysaccharides-An Interdisciplinary Open Access Journal for a Sustainable and Advanced Community
}

\author{
Karin Stana Kleinschek ${ }^{+}(\mathbb{D}$
}

Head of the Institute for Chemistry and Technology of Biobased Systems, Faculty of Technical Chemistry, Chemical and Process Engineering, Biotechnology, Graz University of Technology, Stremayrgasse 9, 8010 Graz, Austria; karin.stanakleinschek@tugraz.at

† Founding Editor-in-Chief of Polysaccharides; Vice president for research of European Polysaccharide network of excellence EPNOE.

Received: 8 June 2020; Accepted: 8 June 2020; Published: 11 June 2020

check for updates

The journal "Polysaccharides" is a new open access journal which has attracted an ambitious editorial team, active in different areas of biopolymer-i.e., polysaccharide-research. As the founding editor-in-chief, it is my wish that the journal will play an important role in the basic research of biopolymers. I strongly believe that polysaccharides and their derivatives are of tremendous importance for a sustainable future.

Access to scientific data is another significant issue today that contributes to development and that will enable the new journal to build bridges between different fields of research.

We aim for a better understanding of the properties of polysaccharides and their bio-assembly (biology, biochemistry, molecular biology), their dis-assembly (chemistry, biotechnology) and their re-assembly (medicine, pharmacy, physic, engineering) (Figure 1). "Polysaccharides" should be open to exciting new ideas and insights that will help in a complementary way to understand, characterize and utilize natural processes. It considers the fascinating fact that that the living world easily assembles the most complex structures that can hardly be reproduced in laboratories. We make significant efforts to master the synthesis of chemical products but find it very difficult to imitate that which living organisms seem to master with ease.

\section{Bio Assembly - Dis Assembly - Re Assembly of Biopolymers}

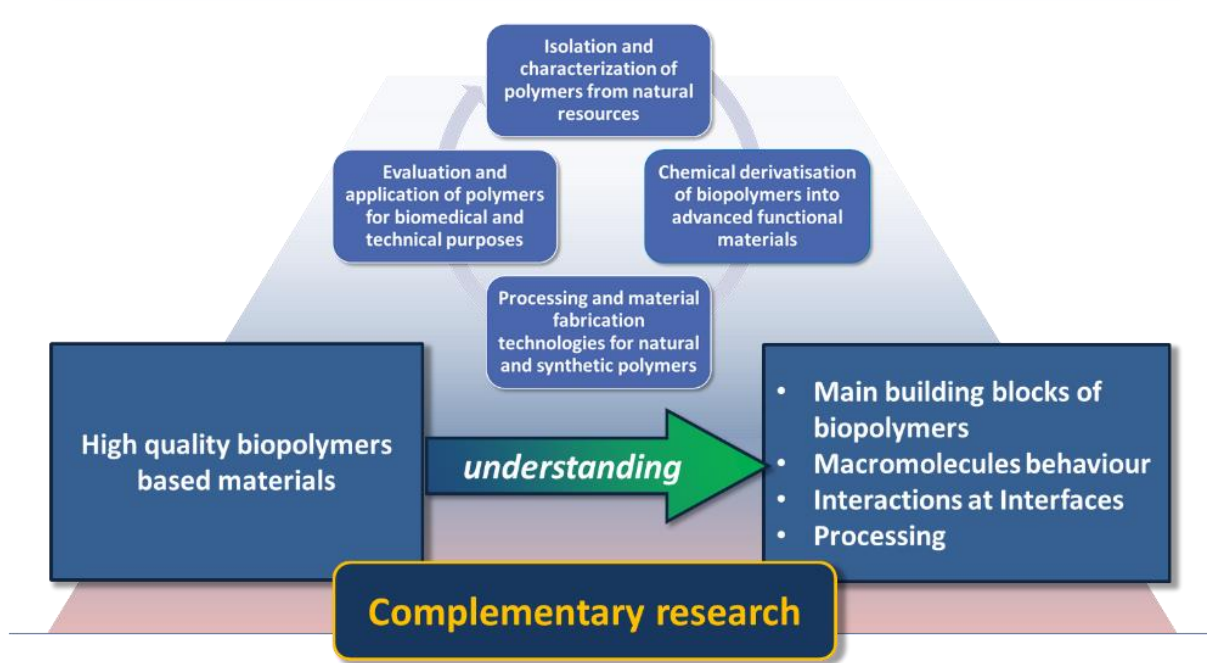

Figure 1. An integrated biopolymer-polysaccharide research concept. 
Polysaccharides and their derivatives are ubiquitous biopolymers and their use has been increasingly explored in previous decades. Polysaccharides are still the largest class of biopolymers used in the classical industries of food, paper, construction and textiles. Their usability in various advanced materials and technologies is becoming more important, also, due to the increased interest for sustainable and functional products and processes. For a focused and increased application of polysaccharides in different biomedical and technical fields, research activities such as the evaluation of the chemical composition, derivatization, characterization and processing are necessary. To apply polysaccharides as functional materials in modern industrial applications, further basic knowledge should be collected and shared among different disciplines.

Thus, the aim of "Polysaccharides" is to highlight the progress and fundamental aspects of new synthesis and derivatization routes, characterization, properties and processing of polysaccharides, all of which are important for their possible application in modern sustainable functional materials and future "green" technologies.

We will be happy to accept the latest results on:

- Advanced methods for the chemical synthesis, derivatization, degradation and extraction of polysaccharides (also oligosaccharides) with an emphasis on regioselectivity and "green" processes (aqueous, enzymatic, (photo)catalytic), avoiding or reducing degradation, waste and yielding chemically well-defined reproducible structures.

- Application of polysaccharides and derivatives or multi-material composites in nutrition and medicine (biomaterials, foods, drugs)

- Processing and shaping of polysaccharide-based materials, either pure or in multi-material blends (e.g., extraction, 3D printing, coating, spinning, extruding, dispersing, precipitating, spraying).

- Critical views on the environmental footprint, sourcing, processing, recycling and (bio)degradation of polysaccharide based materials and their multi-material blends (e.g., food, textiles, packaging, construction) in comparison with existing technologies and with an emphasis on current societal challenges (population and economic growth, ageing, poverty, pollution, mobility, energy, environmental degradation and climate change).

These examples show how interdisciplinary approaches may lead to new knowledge and innovative solutions through a direct interaction between different scientific fields. Thus, we invite you to submit your papers, reviews and short communications to "Polysaccharides" in order to make this journal a success in interdisciplinary research. "Polysaccharides" is an open access journal and will provide rapid publication of scholarly articles. Its mission is to publish cutting edge articles, conference proceedings and Special Issues in order to highlight outstanding research. 